\title{
COMPARTMENTAL RELATIONSHIPS BETWEEN ANURAN PRIMARY SPINAL MOTONEURONS AND SOMITIC MUSCLE FIBERS THAT THEY FIRST INNERVATE ${ }^{1}$
}

\author{
SALLY A. MOODY² AND MARCUS JACOBSON \\ Department of Anatomy, University of Utah School of Medicine, Salt Lake City, Utah 84132
}

Received November 13, 1982; Revised March 21, 1983; Accepted March 21, 1983

\begin{abstract}
The compartmental and clonal relationships between primary motoneurons and the inyotubes they innervate have been studied in Xenopus laevis embryos by initiating clones at blastula stages ( 32 to 512 cells) with intracellular injections of horseradish peroxidase (HRP). Primary motoneurons and ventral myotome belong to the posterior-ventral compartment, whereas sensory neurons and dorsal myotome belong to the posterior-dorsal compartment (Jacobson, M. (1983) J. Neurosci. 3: 1019-1038). The pathways of HRP-labeled primary motor axons, which pioneer the peripheral pathway to the adjacent myotome beginning at stage $21 / 22$, were traced. Within the spinal cord, the axons remained in the posterior-ventral compartment. Upon leaving the cord they were confronted with both dorsal and ventral myotome; they remained almost exclusively in the ventral myotome. At late embryonic stages (40/42) primary motoneurons could be retrogradely labeled by applying HRP to the dorsal myotome, indicating that their axons or a branch had crossed the compartmental boundary.

The primary motor axon also displayed a highly significant preferred association with clonally related myotubes within the ventral myotome. Axon growth only in the compartmentally related myotome and preferred association with clonally related myotubes suggest that the guidance of pioneer axons in the periphery may be based upon factors derived from common ancestry and lineage.
\end{abstract}

Since Harrison (1910) demonstrated that axons grow out of the neural tube without the assistance of preformed Schwann cell chains, the specificity of selection of pathways and targets by axons has been thought to require direct interaction of the axon with the target cell and with the substrate through which it grows. The plethora of possible interactions (e.g., those proposed by Weiss, 1941; Ramón y Cajal, 1960; Gundersen and Barrett, 1979; Singer et al., 1979; Collins and Garrett, 1980; Katz et al., 1980; Letourneau, 1982) suggests that there is no single universal mechanism of axonal guidance but

\footnotetext{
${ }^{1}$ This work was supported by Grant BNS 8116768 from the National Science Foundation and by National Institutes of Health, National Research Service Award NS 06955 from the National Institute of Neurological and Communicative Disorders and Stroke. Statistical analyses were performed by Dr. James C. Reading, Biostatistics Division, Department of Family and Community Medicine, University of Utah School of Medicine. The technical assistance of Hannelore Mueller and the typing assistance of Karen Evans are gratefully acknowledged.

${ }^{2}$ To whom correspondence should be addressed, at Department of Anatomy, University of Virginia, Charlottesville, VA 22908.
}

rather that different mechanisms may operate in different species, different situations, and at different times during development. Recent studies of axonal guidance in insects have revived the concept that "pioneer" axons (Ramón y Cajal, 1908; Harrison, 1910; Speidel, 1933; Weiss, 1941) seek out the first peripheral pathways to appropriate target areas and are used as substrates by later differentiating axons (Bate, 1976; Bentley and Keshishian, 1982; Edwards, 1982; Goodman et al., 1982). 'The existence of similar pioneer axons has not been demonstrated in vertebrates, but it has been suggested that anuran Rohon-Beard axons pioneer the dorsal spinal sensory tract (Katz and Lasek, 1978, 1979), anuran primary motor axons, which first innervate somitic musculature, provide a pathway for subsequent limb motor axons (Taylor, 1943), and mouse and chick commissural neurons pioneer the contralateral basolateral spinal tract (Holley, 1982; Holley et al., 1982).

Pioneer axons must themselves have a mechanism to guide them to appropriate targets. Recent studies in holometabolous insects have suggested that axon pathways to CNS targets may be determined genetically 
(Garcia-Bellido, 1975; Lawrence, 1975, 1978; Ghysen and Deak, 1978; Vandervorst and Ghysen, 1980). By initiating clones mutagenically early in development it has been demonstrated that body segments and appendages in the adult are subdivided into sharply defined compartments which are derived from groups of embryonic founder cells in the imaginal discs (Garcia-Bellido, 1975; Garcia-Bellido et al., 1973, 1976; Crick and Lawrence, 1975; Morata and Lawrence, 1977, 1978). It has been suggested that growth of peripheral axons may be restricted to their own compartment en route to the CNS, and that perhaps these axons are compartmentally related to their CNS targets (Lawrence, 1978). However, in certain mutants of Drosophila, sensory axons can cross compartmental boundaries (Anderson, 1981; Palka et al., 1981; Palka, 1982).

Clonal analysis of cell lineages has been extended to a vertebrate embryo, Xenopus laevis, by injecting a marker protein into individual blastomeres and tracing the phenotypic and spatial distribution of the labeled descendants (Jacobson and Hirose, 1978, 1981; Hirose and Jacobson, 1979; Jacobson, 1982, 1983). These studies have shown that specific embryonic body regions, called compartments by analogy with those of insects, are populated by the progeny of specific groups of ancestral cells in the 512- to 1024-cell stage blastula (stage 8-8+ of Nieuwkoop and Faber, 1967). Seven ancestral cell groups give rise to seven compartments: one anterior-ventral (AV), two anterior-dorsal (AD), two posterior-ventral (PV), and two posterior-dorsal (PD) as described by Jacobson (1982). In the trunk region, dorsal spinal cord and dorsal myotome are in the posterior-dorsal compartment and are descended from the posterior-lateral (PL) ancestral cell group. Likewise, ventral spinal cord and ventral myotome, in the posterior-ventral compartment, are descended from the posterior-medial (PM) ancestral cell group (Jacobson, 1983).

We have observed that when the axon of a primary spinal motoneuron leaves the neural tube, it is confronted with myotome in both posterior-dorsal and posterior-ventral compartments. The present report demonstrates that, although the outgrowing primary motor axon is confronted with mesodermal cells belonging to two different compartments, it chooses to grow almost exclusively within the mesodermal tissues of its own compartment (PV). We also show that primary motor axons preferentially associate with clonally related myotubes within the PV compartment. This evidence suggests that compartmental relationships may aid in confining pioneer axon growth within certain tissue boundaries, and that clonal relationships may aid in guiding the pioneer axon along its appropriate pathway.

\section{Materials and Methods}

Procedures. $X$. laevis fertilized eggs were obtained by gonadotropin-induced matings of adult frogs. Jelly coats were removed from eggs by washing in $5 \mathrm{mM}$ dithiothreitol buffered with 0.05 M HEPES, pH 8.9. After three washes in $100 \%$ Steinberg's solution, embryos with prominent pigment gradients and with regular cleavage patterns were selected (Jacobson, 1981). One blastomere of each embryo was injected with concentrated aqueous horseradish peroxidase (HRP, Sigma type IX) as described previously (Hirose and Jacobson, 1979; Jacobson and Hirose, 1981). Injected embryos were placed in a $20^{\circ} \mathrm{C}$ incubator to resume development. They were fixed by immersion in $2.5 \%$ glutaraldehyde, $1 \%$ paraformaldehyde in $0.1 \mathrm{M}$ phosphate buffer, $\mathrm{pH} 7.4$, serially sectioned at $24 \mu \mathrm{m}$ with a cryotome, and histochemically processed for the HRP reaction product as previously described (Hirose and Jacobson, 1979; Jacobson and Hirose, 1981).

Definition of the compartmental boundary in the myotome. The ventral part of the myotome and the ventral part of the spinal cord, including primary motoneurons, both lie in the PV compartment and share a common ancestry from cells of the posterior-medial (PM) ancestral cell group of the 512-cell embryo; the dorsal part of the myotome and the dorsal part of the spinal cord, both in the $\mathrm{PD}$ compartment, are both descended from the PL ancestral cell group (Jacobson, 1983). To reconstruct the compartmental boundary in the myotome, several blastomeres in both the PM and PL ancestral cell groups (one blastomere per embryo) were injected with HRP at the 512-cell stage. Camera lucida drawings of HRPlabeled myotubes in each of these embryos were made. Ten serial drawings at four different axial levels from each embryo were superimposed. The position of the compartmental boundary is defined as the boundary between the region containing labeled myotubes and the totally unlabeled region of myotome.

Identification of primary motoneurons. Primary motoneurons are the largest cells in Xenopus embryonic ventral plate and are among the earliest to cease mitosis (Lamborghini, 1980) and to differentiate (Coghill, 1913; Hughes, 1959). They can be identified by their large somas (10 to $20 \mu \mathrm{m}$ ) at the margin of the white matter and their extensive dendritic arborization in the lateral funiculus (Blight, 1978). Those cells identified as primary motoneurons in the present report are analogous to those previously reported in Triturus (Blight, 1978) and Xenopus (Hughes, 1959; Roberts et al., 1981).

Retrograde labeling. The following experiments were performed in order to determine whether primary motor axons innervate the entire myotome or only the portion of the myotome to which they are compartmentally related. Embryos at stages 29 to 32 or 37 to 40 were placed in a well of modeling clay in a Petri dish. Most of the Steinberg's solution was removed from the trunk region, drying the ectoderm overlying the postotic somites. A tungsten needle with tip diameter of 10 to $20 \mu \mathrm{m}$, coated with crystalline HRP, was lowered into the superficial region of one somite; somites 5 through 10 were used. A small puncture was made in either the dorsal or ventral part of the myotome, and the needle was left in place for 15 to $30 \mathrm{sec}$ while the HRP dissolved. After removing the needle the embryo was placed in $50 \%$ Steinberg's solution. Embryos were fixed 6 to $24 \mathrm{hr}$ after HRP application (at stages 34 and 40 to 42 ), sectioned, and reacted for the HRP product as described above. Camera lucida drawings were made to determine the extent of the myotome exposed to HRP and to mark the position of the retrogradely labeled neurons in the CNS.

Labeling of primary motoneurons and their pioneer 
axons. The following experiments were performed to describe the primary motor axon's course within the myotome and to define its relationship to the compartmental boundary in the myotome and to the particular myotubes with which it was clonally related. Embryos were selected as described above, and the blastomeres which gave rise to primary motoneurons were injected with HRP at the 32- through 512-cell stage. Embryos were fixed at stages 24 to 42 and processed as above. Motor axons, which were filled with HRP by this method, were drawn to determine whether axons exiting from the neural tube grew in the PD or PV compartment.

Additionally, the number of myotubes that were clonally related to the axons (i.e., descended from the same blastomcre and thus also labeled with HRP) were counted in the same transverse section as a labeled axon. Since myotubes extend the entire length of the Xenopus myotome (Hamilton, 1969), this count included all labeled myotubes in one segment. Using an eyepiece graticule, the labeled motor axon was centered in $\times 800$ field. All myotubes within a $10-\mu \mathrm{m}$ zone on either side of the axon and within the $24-\mu \mathrm{m}$ thick section were counted, and the number of these myotubes that were labeled with HRP was tabulated. The $20-\mu \mathrm{m}$ zone surrounding the axon was chosen to give a conservative estimate of the number of myotubes that might be contacted by filopodia, which we cannot visualize with our histochemical methods. The numbers of labeled and nonlabeled myotubes within the $20-\mu \mathrm{m}$ zone (presumed "contacted") were compared to the numbers of labeled and nonlabeled myotubes outside that zone (presumed "not contacted") by performing Mantel-Haenszel $\chi 2$ tests (MHC; Mantel and Haenszel, 1959; Mantel, 1963). The MHC test compared the observed number of "contacted" labeled myotubes to an expected value derived from the proportion of labeled and nonlabeled myotubes outside the $20-\mu \mathrm{m}$ zone and calculated a $\chi^{2}$ value with 1 degree of freedom. A MHC test was done for each myotome containing a labeled axon, then a summary MHC test was calculated for each embryo, and finally a summary MHC test over all embryos was performed to obtain the overall $p$ value. This procedure was done on the assumption that there are 150 myotubes in the segmented Xenopus myotome (Blackshaw and Warner, 1976).

\section{Results}

Compartmental boundaries. In the trunk region of a stage $31 / 32$ embryo the ventral plate of the spinal cord, mesenchymal cells of the sclerotome, the notochord, and the ventral myotome were all descended from the PM ancestral cell group and thus were constituents of the PV compartment. The dorsal plate of the spinal cord and the dorsal myotome were descended from the PL ancestral cell group and thus belonged to the PD compartment (Jacobson, 1982, 1983). Figure 1 illustrates that at approximately the horizontal plane extending from the ventral limit of the central canal, myotube progeny from either the PM or PL ancestral cell group were separated. This region of the myotome was consistently seen as a spatial boundary between the two myotube lineages and, as such, was considered a compartmental boundary. $\mathrm{Be}-$ cause the clones generated from one blastomere at the 512-cell stage were small, compartmental boundaries rarely were precisely outlined by a row of labeled cells. And because of individual variations in blastomere cleavage planes and in coherent clonal growth, accurate labeling of an entire ancestral cell group in a single embryo, in order to label an entire myotome compartment, was not feasible (Jacobson, 1983). Indeed, such variations could alter the precise placement of these boundaries. Nonetheless, the myotome boundary generally coincided with the region from which the primary motor axon exited from the neural tube. Thus, this axon may enter either the PV compartment (ventral myotome, sclerotome, and notochord) or the PD compartment (dorsal myotome).

Retrograde labeling. Embryos in which the detectable diffusion of HRP extended across the dorsoventral myotome boundary contained labeled primary motoneurons, Rohon-Beard neurons, and extramedullary neurons. These embryos were discarded. Primary motoneurons were retrogradely labeled when HRP was applied to the ventral myotome at all stages (Fig. 2). In many cases ( 8 of 12 ) the epidermis was heavily labeled from the ventral HRP application, and Rohon-Beard and extramedullary neurons were retrogradely labeled as their axons coursed ventral to the injection site in the space between myotome and epidermis (Fig. 3). When HRP was applied to the dorsal myotome it was retrogradely transported only by Rohon-Beard and extramedullary neurons in embryos receiving the HRP at stages 32 to 38 and killed at stages 39 to 40 (Fig. 4). However, in embryos receiving $\mathrm{HRP}$ at stages $40 / 41$ and killed at stage 42, Rohon-Beard neurons, extramedullary neurons, and primary motoneurons were labeled (Fig. 5). By these stages either a branch from the primary motor axon had entered the dorsal myotome or a secondary population of primary motoneurons had innervated the dorsal myotome.

Primary motor axon compartmental associations. During the primary motor axon's intracentral course, as it traveled caudally in the basolateral tract, it remained within the PV compartment. Upon leaving the spinal cord the axon was confronted with mesenchymal cells of the sclerotome (PV), notochord (PV), and myotubes of the dorsal (PD) and ventral (PV) myotome. The axon entered the myotome in the region where myotube lineages from different ancestral cell groups were segregated (Figs. 1 and 6), growing across sclerotome cells before entering the myotome in older embryos (Fig. 7). Growth within the myotome was confined to the PV compartment (approximately 150 to $200 \mu \mathrm{m}$ ). Although the compartmental boundary in the myotome could not be precisely identified in any one embryo, primary motor axons were never found more that $15 \mu \mathrm{m}$ dorsal to the horizontal plane extending from the ventral limit of the central canal (Fig. 6). Once within the ventral myotome, the axon stereotypically grew ventrally within the medial region of the myotome, giving off small branches among myotubes (Fig. 8). At stages 32 to 33, profuse arborization among medial and lateral myotubes began (Fig. 9).

Primary motor axon clonal associations. The entire ventral myotome was not filled with labeled myotubes at any of the injection ages. Rather, clusters of labeled 
myotubes extended across the width of the myotome at the level of the notochord (Figs. 7 to 10). The mean number of labeled myotubes was 8 , but as many as 39 were labeled in some cases. In its course, the primary motor axon closely associated with many of the labeled myotubes (Figs. 6, 8, and 9). In a few embryos (7 of 195) that were injected at the 128-cell stage and later, there were no labeled myotubes in the myotome containing a

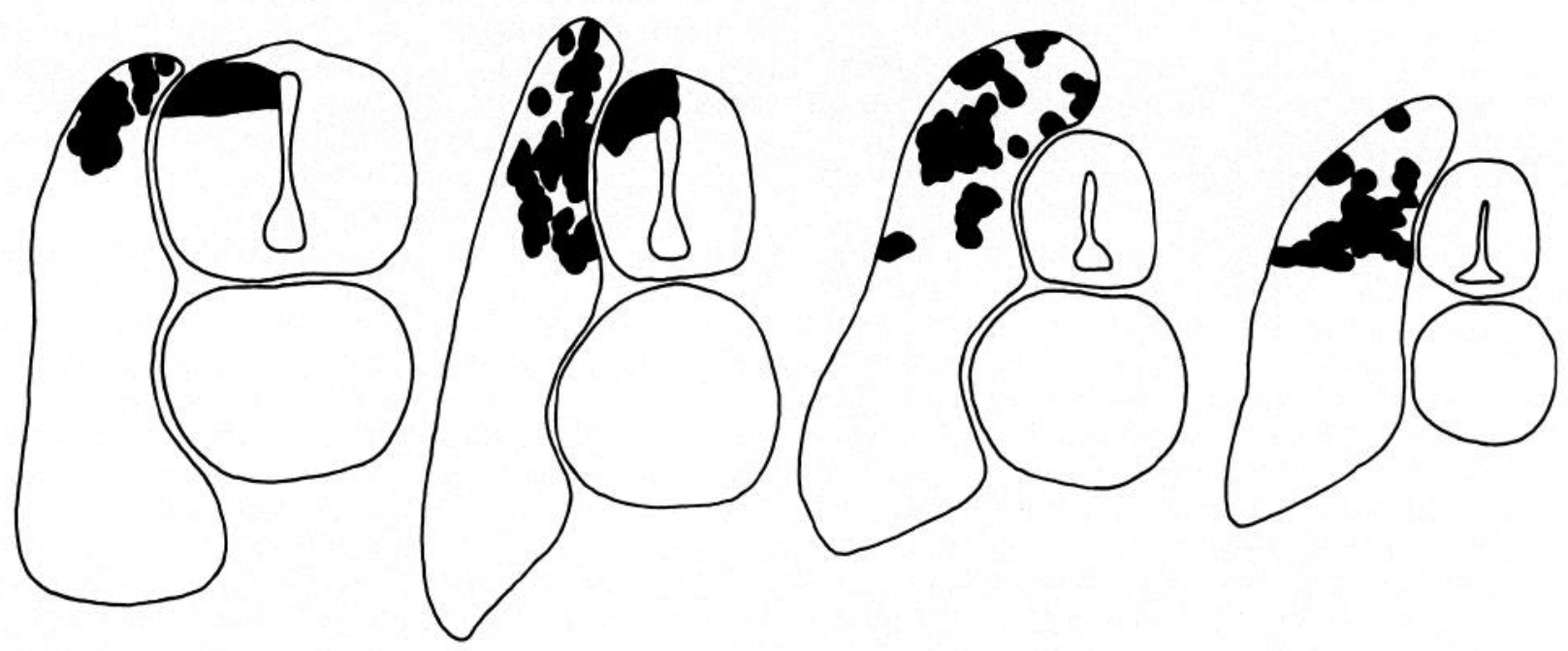

1A

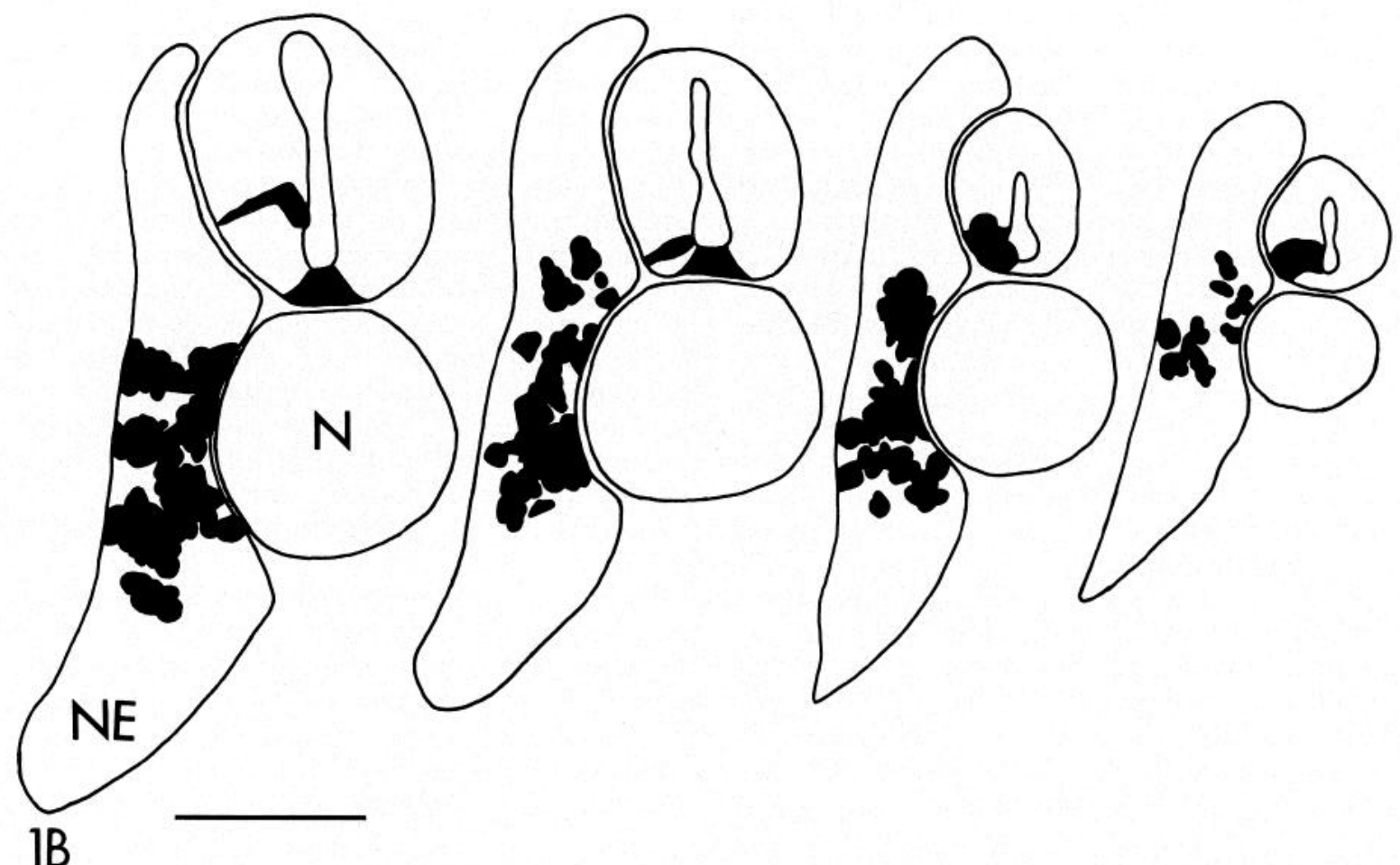

Figure 1. Camera lucida drawings of labeled (black) cells after HRP injection of a blastomere at the 512-cell stage illustrate the separation between myotome regions descended from different ancestral cell groups. Although a distinct boundary cannot be drawn, the domains of the two lineages generally meet at a horizontal plane drawn from the ventral limit of the central canal. $A$, The injected blastomere was within the posterior-lateral ancestral cell group. Its descendants were within the posterior-dorsal compartment. Labeled myotubes extended from the dorsal tip of the myotome to the level of ventral limit of the central canal. Bar equals $100 \mu \mathrm{m}$. B, The injected blastomere was within the posterior-medial ancestral cell group. Its descendants were within the posterior-ventral compartment. Labeled myotubes extended from the level of the ventral limit of the central canal to the dorsal portion of the nephrotome $(N E)$. The notochord $(N)$ was labeled in this embryo but was not blackened in the drawing. Bar equals $100 \mu \mathrm{m}$. 
labeled axon, although at other segmental levels myotubes were labeled.

All axons analyzed ( $N=195$ from 49 embryos) were divided into two categories. Short axons (those with less than 10 myotubes, both labeled and unlabeled, within the $20-\mu \mathrm{m}$ zone) were not statistically analyzed. Many of those were only 10 to $20 \mu \mathrm{m}$ in length and terminated in a growth cone (Figs. 7 and 10). Although labeled filopodia could not be discerned in our preparations, labeled growth cones appeared as though oriented toward labeled myotubes (Figs. 7 and 10). In trunk regions where myotomes had segmented, labeled myotubes generally were no more than 30 to $50 \mu \mathrm{m}$ from where the labeled primary motor axons grew out of the neural tube (Figs. 7 and 10). In the tail bud, where the myotomes were unsegmented, short axons extending from the neural tube were usually within 10 to $30 \mu \mathrm{m}$ of labeled myotubes (Fig. 11). Some labeled myotubes were adjacent to the radial cells of the ventral plate that would probably differentiate into primary motoneurons (Fig. 10A).

Long axons (those with more than 10 myotubes within the $20-\mu \mathrm{m}$ zone) were included in the statistical analysis because they were considered to have established most of their peripheral pathway. As these axons grew through the ventral myotome, they associated with a large percentage of the labeled myotubes (Figs. 6, 8, and 9). Although many nonlabeled myotubes were within the $20-\mu \mathrm{m}$ zone surrounding the axon, the number of labeled myotubes within that zone was much greater than expected based on the ratio of labeled to unlabeled myatubes in the entire myotome (Table I). A MHC test was performed for each axon $(N=123)$ contacting more than 10 myotubes, and a summary MHC test was calculated for each embryo $(N=36)$. These tests and the summary MHC test for the entire population of embryos were highly significant $(p<0.001)$, indicating a preferred association of labeled axon with labeled myotubes within the ventral myotome (Table I).

\section{Discussion}

Amphibian primary spinal motoneurons are among the earliest neurons to be born (Lamborghini, 1980). They extend the first axons into the myotome (Coghill, 1913; Hughes, 1959; Muntz, 1964; Kullberg et al., 1977; Blight, 1978) and mediate the initial embryonic trunk flexures (Coghill, 1913; Muntz, 1975; Roberts et al., 1981) and later larval swimming activity (Stehouwer and Farel, 1980; Forehand and Farel, 1982). Their precocious development has allowed study of the establishment of pioneer axon-target relationships. We propose that the initial pattern of axon growth is determined by the axon's ability to recognize tissues to which it is compartmentally related. Furthermore, its specific pathway may be influenced by the spatial distribution of clonally related myotubes. The primary motor axon did not seem to be growing randomly among a homogeneous population of myotubes but appeared initially to confine its growth to the myotubes in the appropriate compartment and to choose its peripheral pathway by preferentially associating with myotubes to which it was clonally related. This pioneer axon pathway within the myotome may provide the frame for future axon growth (to limb, hypaxial, and epaxial musculature) during later differentiation.

Clonal analyses have demonstrated that the ancestral cell groups which are established in the 512-cell stage Xenopus embryo each give rise to regions (compartments) of CNS, epidermis, mesoderm, and endoderm (Jacobson, 1980, 1983). We have illustrated that the embryonic myotome, although appearing histologically homogeneous, can be divided into two compartments which are derived from different ancestral cell groups. The compartmental boundary zone in the myotome, which is not strictly defined by a corresponding morphological landmark, is located at the point from which primary motor axons leave the spinal cord. This fortuitous placement allowed us to test whether primary motor axons preferentially chose to grow within a particular compartment.

Axon growth within compartments. Experiments on holometabolous insects have suggested that peripheral sensory axons may grow only within their own compartment, be deflected by compartmental boundaries, and affiliate only with compartmentally related neurons in the CNS (Lawrence, 1975; 1978; Ghysen and Deak, 1978; Ghysen, 1980; Vandervorst and Ghysen, 1980). However, compartmental affiliation cannot be the only mechanism guiding axons because sensory axons displaced by mutations cross compartmental boundaries (Deak, 1978; Palka et al., 1981), and sensory axons from transformed appendages make CNS connections appropriate for the original compartmentally related neurons in the CNS but not appropriate for the transformed tissue (Ghysen, 1978; Palka et al., 1979; Anderson, 1981; Stocker and Lawrence, 1981; Palka, 1982).

Our experiments have demonstrated that primary motor axons grew within the myotome in a specified compartment. Although we could not determine whether the primary motor axon crossed the compartmental boundary, as this boundary cannot be precisely defined in any individual embryo with our techniques (Jacobson, 1983), nonetheless, the primary motor axon probably had filo-

Figure 2. Retrogradely labeled primary motoneuron $(M)$ after HRP application (stage 38 , killed stage 39 ) to the ventral myotome. 'The extent of the injection site in adjacent sections is bracketed. 'I'his tissue has not been counterstained; HRPlabeled cells ( $\mathrm{M}$ and myotubes in bracketed area) and melanocytes (arrowheads) appear black in this and following micrographs. $G$, gut; $N$, notochord; $P D$ compartment in the dorsal myotome; $P V$ compartment in the ventral myotome. Magnification $\times 275$.

Figure 3. Retrogradely labeled primary motoneuron $(M)$ and Rohon-Beard neuron $(R)$ after HRP application (stage 40, killed stage 41 ) to the ventral myotome and epidermis $(E)$. The Rohon-Beard cell transported the HRP because its axon (not visible at this magnification) was between the myotome and epidermis, innervating labeled epidermal cells. Magnification $\times 300$.

Figure 4. Retrogradely labeled Rohon-Beard neuron $(R)$ after HRP application (stage 38 , killed stage 40 ) to the dorsal myotome. Magnification $\times 300$.

Figure 5. Retrogradely labeled Rohon-Beard neuron $(R)$ and primary motoneuron $(M)$ after HRP application (stage 41, killed stage 42 ) to the dorsal myotome. Note the dorsal motor axon $(A)$ that invaded the dorsal myotome. Magnification $\times 300$. 

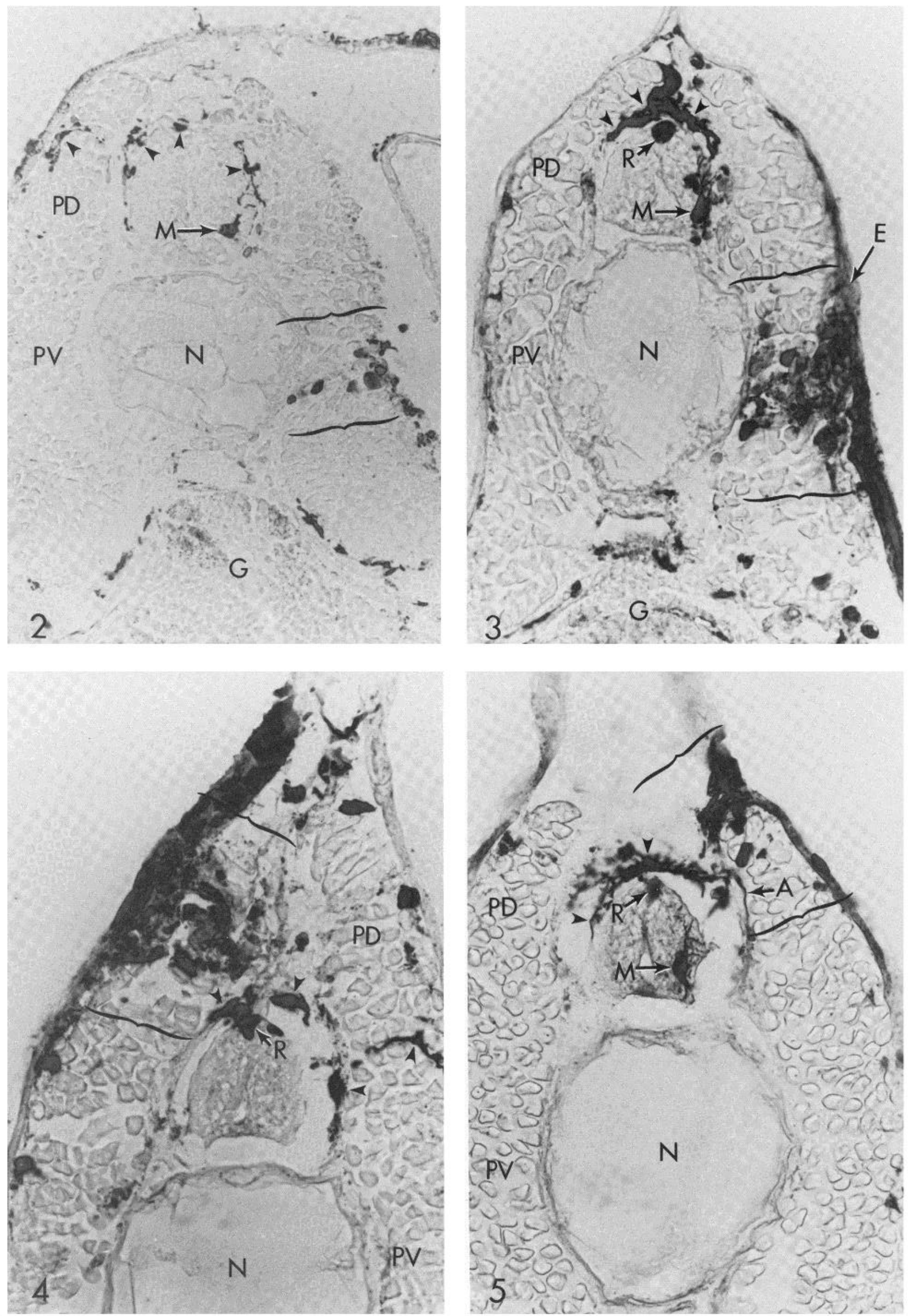

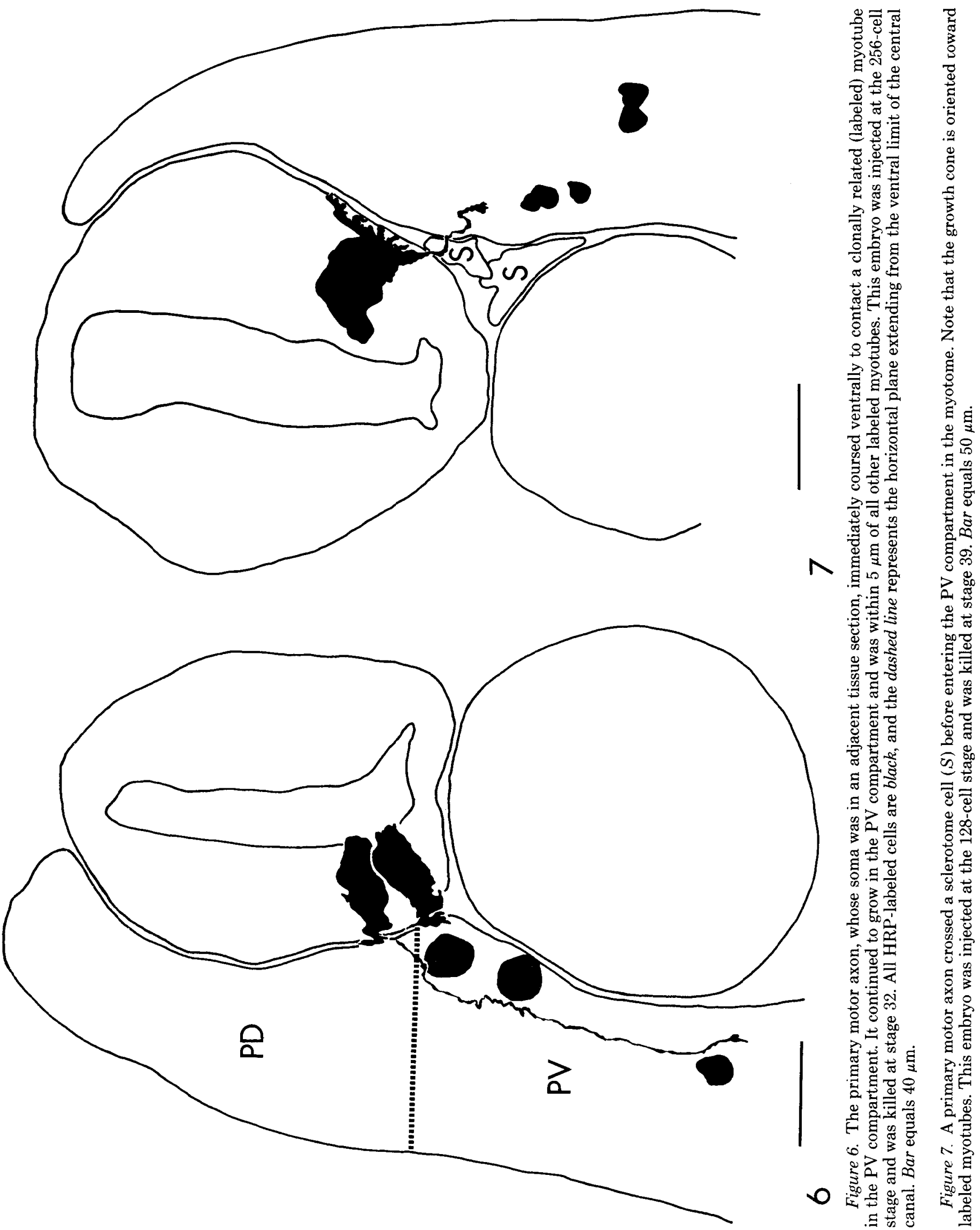


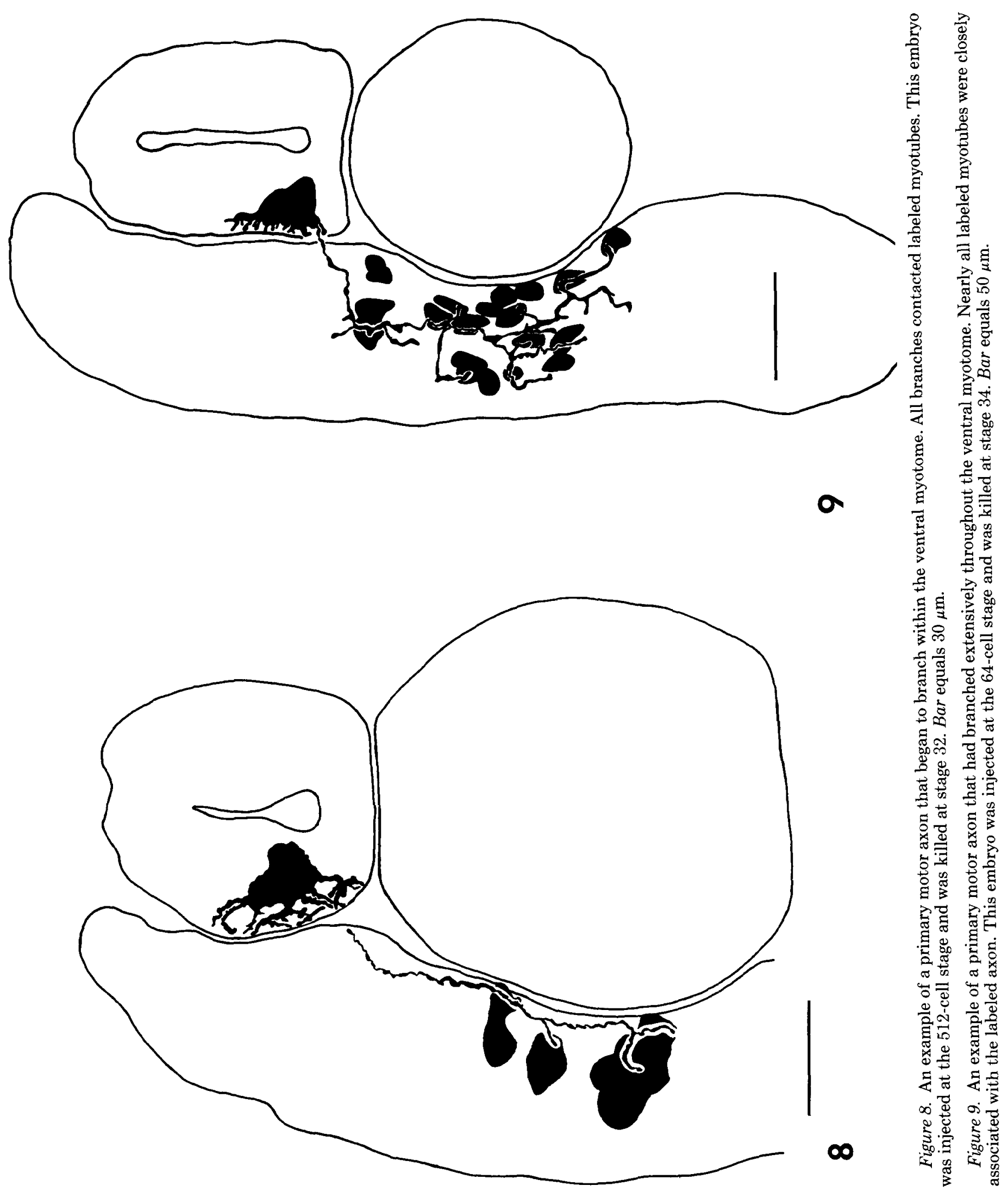



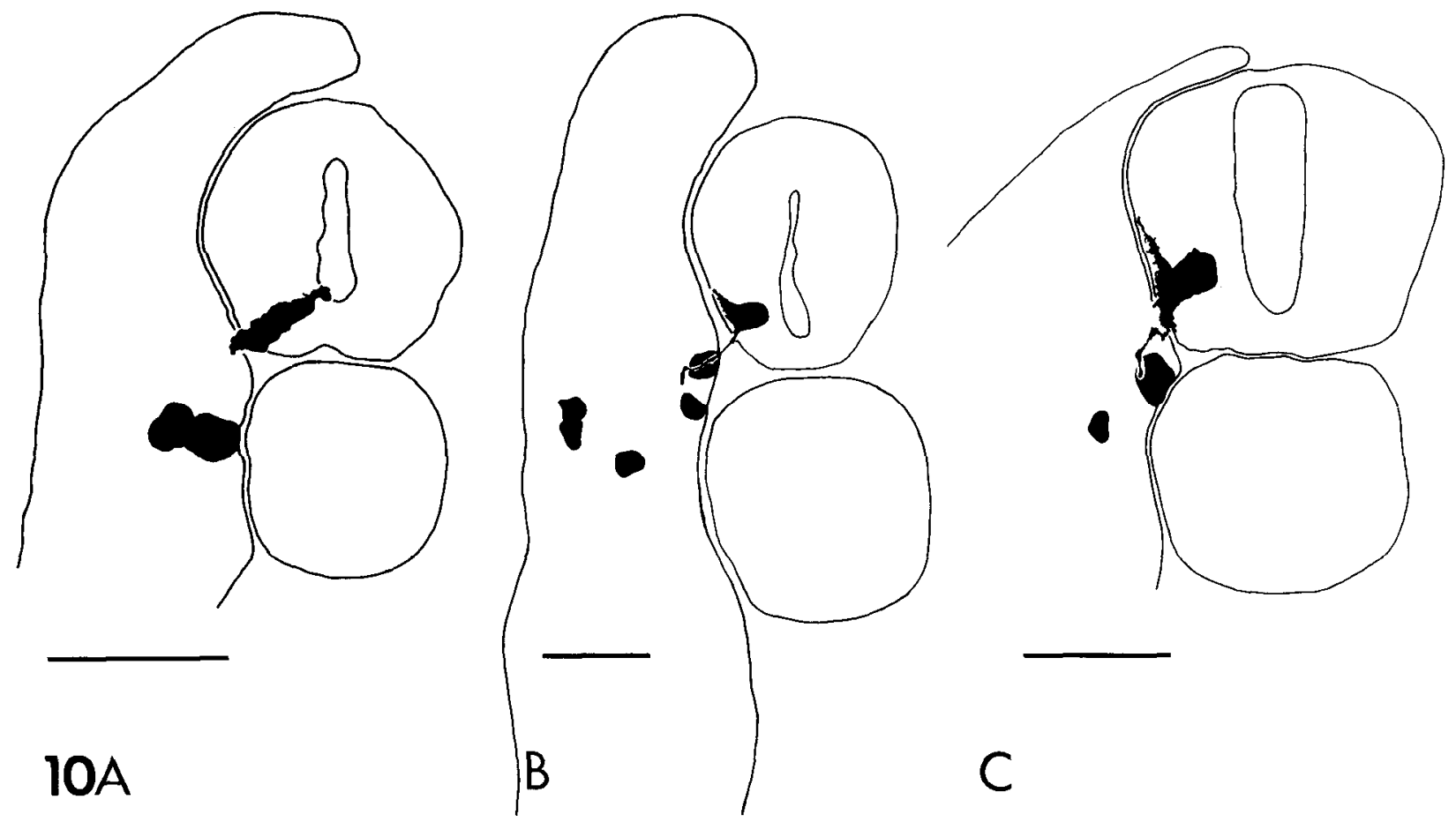

$10 \mathrm{~A}$

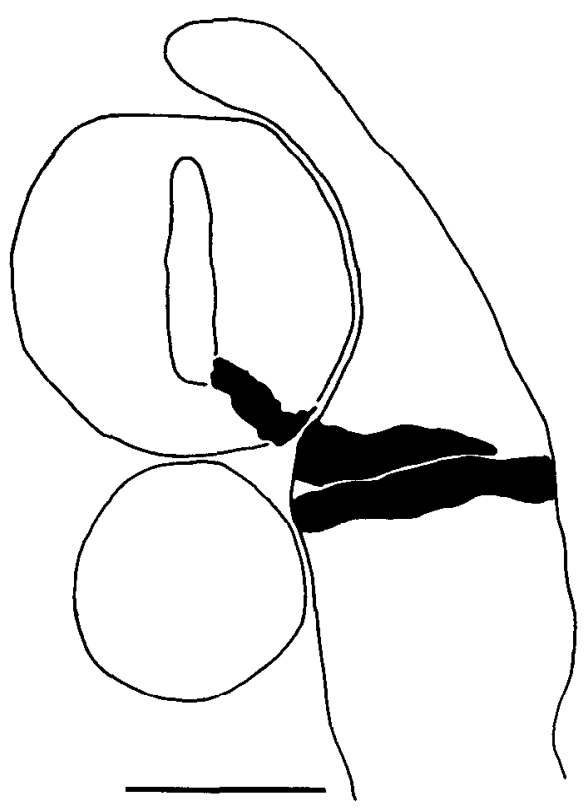

$11 \mathrm{~A}$

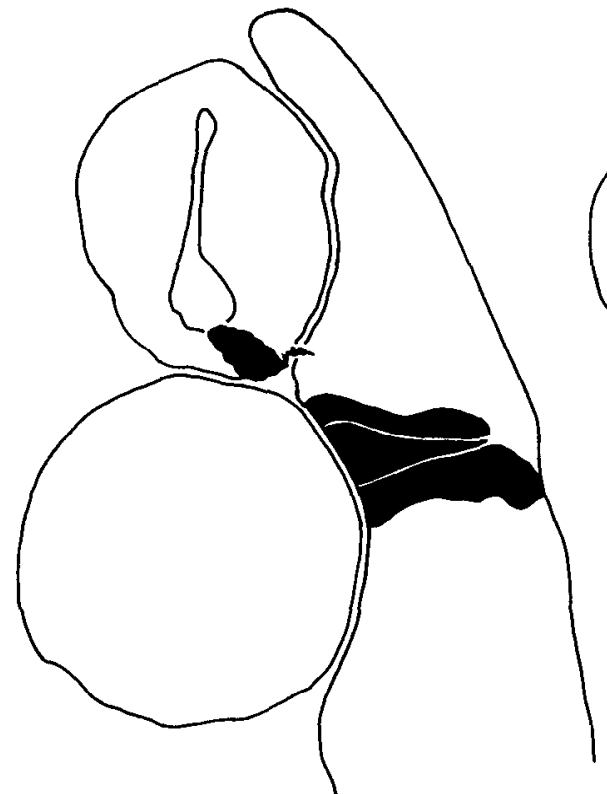

B

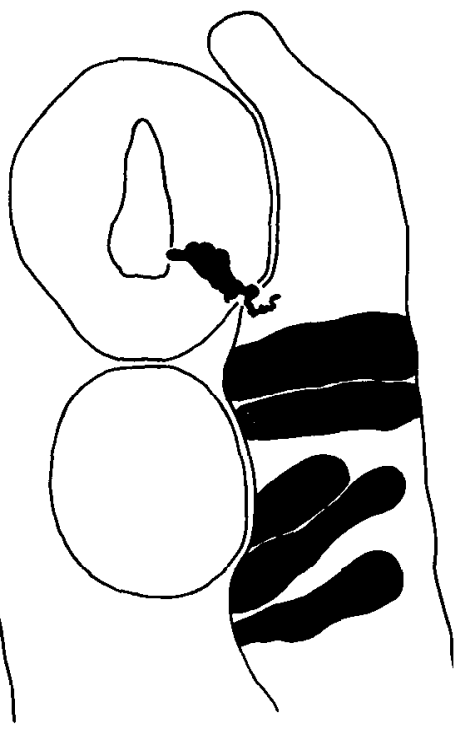

C

Figure 10. Three examples of short primary motor axons from the trunk region where somites had segmented. The labeled myotubes generally were within 30 to $50 \mu \mathrm{m}$ from the neural tube. Axons occasionally contacted the labeled myotubes soon after leaving the neural tube ( $B$ and $C$ ). All three embryos were injected at the 256-cell stage and were killed at stage 32 . Bars equal $40 \mu \mathrm{m}$.

Figure 11. Three examples of undifferentiated primary motoneurons in the tail bud, where the somites had not segmented. Bar equals $40 \mu \mathrm{m}$. A, Labeled myotubes were adjacent to the neural tube region from which the primary motor axon would leave. The labeled radial cell in the ventral plate may have been an undifferentiated primary motoneuron. This embryo was injected at the 128-cell stage and was killed at stage $32 . \mathrm{B}$, Labeled myotubes were within $20 \mu \mathrm{m}$ of the labeled short primary motor axon. This embryo was injected at the 256 -cell stage and was killed at stage 32 . $C$, Labeled myotubes were within $10 \mu \mathrm{m}$ of the labeled short primary motor axon. This embryo was injecled at the 256 -cell stage and was killed at stage 32. 
podial access to myotubes in both PV and PD compartments when it left the neural tube. It is significant that the primary motor axon needed only to cross a 7- $\mu \mathrm{m}$ gap to reach the myotome (Nordlander et al., 1982). In every case, the axon was seen to associate with myotubes in the PV compartment.

The segregation of the myotome into two populations and the selective innervation of those populations further suggest that primary motor axon pathways in the myotome are based initially on compartmental affiliations. This segregation was demonstrated in our experiments by the fact that primary motoneuron somas were not retrogradely labeled by application of HRP to the dorsal myotome before stage 40 . Although primary motor axons eventually grew throughout the myotome, their initial growth was restricted to the ventral myotome. Thus, initial innervation was restricted to the compartment to which the axon also belonged. One should also consider whether initial restriction of primary motor axons to the ventral myotome might be due to technical shortcomings.
For example, the HRP could have been rapidly washed away in our aqueous preparations, making it unavailable for uptake by primary motor axons in the dorsal myotome. However, Rohon-Beard and extramedullary neurons were heavily labeled by our methods. Another possibility is that survival times may not have been sufficient for retrograde transport from dorsal myotome to primary motoneuron. However, we have obtained light labeling of primary motoneurons after $6 \mathrm{hr}$ and heavy labeling after 18 to $24 \mathrm{hr}$. Similar labeling intensities have been obtained in other studies by using 48 -hr survival periods (Farel and Bemelmans, 1980; Forehand and Farel, 1982). We preferred the shorter survival times (12 to $24 \mathrm{hr}$ ) because we could more accurately determine the time of dorsal motor axon growth into the dorsal myotome (stages 40 to 42 ).

The growth of a primary motor axon into the dorsal myotome is an interesting event because it represents a time in development when the axon crosses a compartmental boundary. Our material did not reveal whether

TABLE I

Mantel-Haenszel $\chi^{2}$ values calculated for association of primary motor axon with clonally related myotubes within a 20- $\mu m$ zone

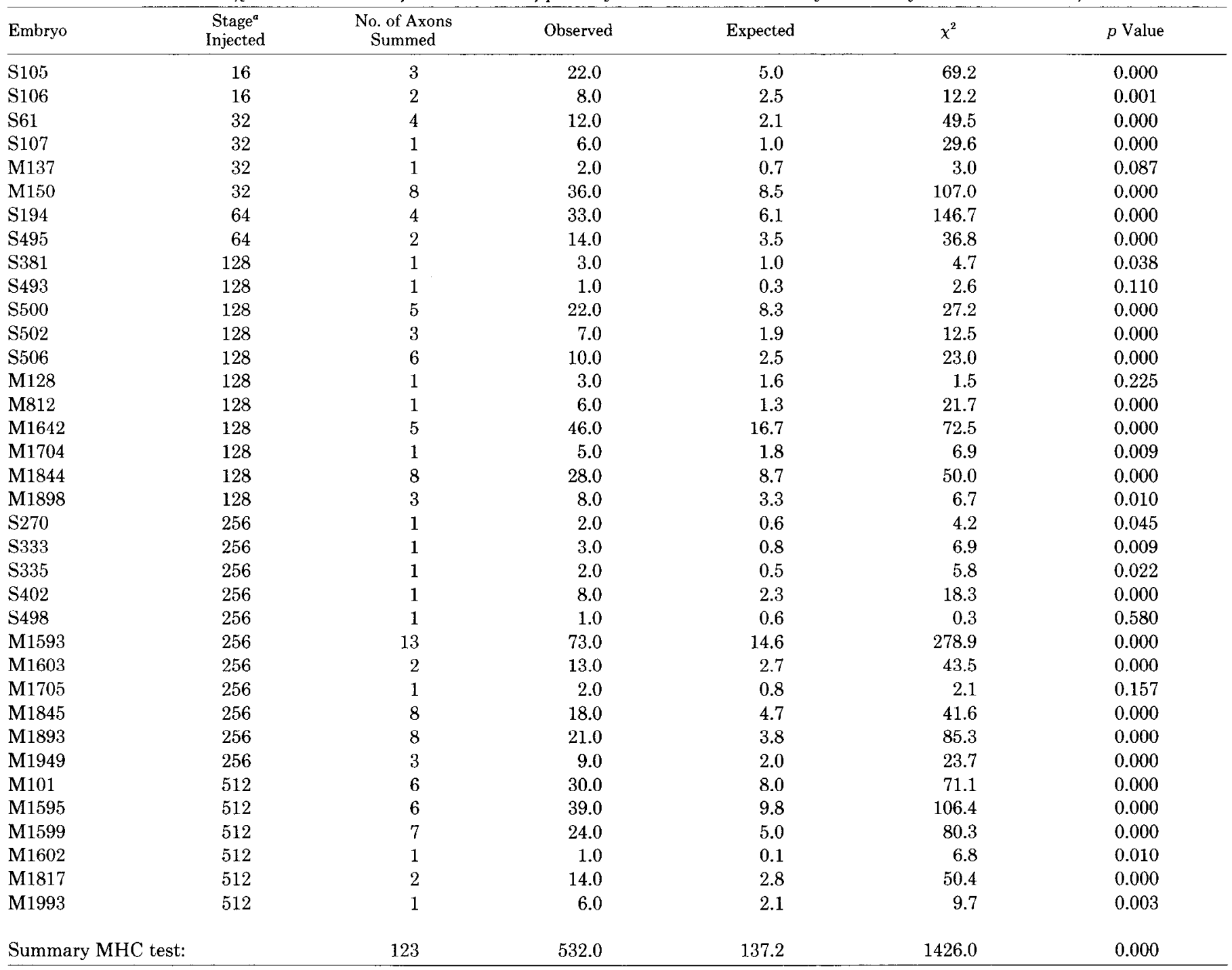

${ }^{a}$ Stage $=$ number of cells in embryo at time when one blastomere was injected. 
the dorsal axon was a branch from the earlier growing ventral primary motor axon or was the axon of a secondary population of motoneurons with soma morphology and location identical to those of primary motoneurons. Ventral axons were not visualized after HRP application to dorsal myotome, whereas dorsal axons were (Fig. 5). This does not mean that dorsal axons were not branches; the HRP may not have anterogradely filled the ventral branch. We also could not distinguish whether dorsal axons were branches in our clonally labeled preparations. In those few cases where dorsal axons were observed, the clones had been initiated early (32- to 128-cell stages), axons were in bundles, and the precise origin of the dorsal axons could not be traced because of the overlapping HRP-labeled profiles. Nonetheless, the fact that motoneurons innervate dorsal myotome after ventral primary motor axons arborize throughout the ventral myotome suggests that axon confinement to peripheral compartments may be effective only during the early stages of pioneer axon outgrowth. Compartmental boundaries may no longer restrict axon pathways later in development (Jacobson, 1980).

It is possible that primary motor axons are determined to grow in the observed pathways regardless of the lineage characteristics of the surrounding tissues. The confinement of primary motor axons to their appropriate compartments could be fortuitous and have no bearing on the mechanisms that guide the axons. Palka et al. (1981) have reported that when particular mutations cause anterior compartment wing hairs to develop in the posterior wing compartment in Drosophila, not all sensory axons reunited with the nearby anterior compartment wing veins; axons proximal to the anterior cross vein chose aberrant, noncompartmentally related pathways to reach the CNS. This experiment shows either that factors other than compartmental relations direct axon pathways or that the aberrant axons were not pioneers and thus did not recognize compartmental affiliations. Indeed, in our experiments the earliest axons confined their growth to compartmentally related myotome, but later axons could grow into noncompartmentally related myotome.

It should be remembered that axon pathway confinement based upon compartmental membership certainly is not universal. Spinal commissural neurons belong to the PD compartment, but their axons arborize among neurons in the PV compartment. Mauthner neurons, which originate in the $\mathrm{AD}$ compartment, have axons in the PV compartment that synapse upon primary motoneurons (Jacobson, 1983). Possibly the connections made within the CNS are established without reference to clonal origins, whereas the guidance of initial peripheral axons may rely upon compartmental restrictions.

Axon association with clonally related myotubes. In regions of unsegmented myotome, a clonally related myotube might be directly adjacent to or within 30 to $50 \mu \mathrm{m}$ of the neural tube. In regions of segmented myotome, usually one of the first 10 myotubes along the pathway of the primary motor axon was clonally related. There was a highly statistically significant association of primary motor axons with clonally related myotubes along their entire peripheral pathway. This preference suggests that axonal guidance may be based on specific recognition between axons and specialized, clonally related cells in their pathway, possibly in a manner similar to that described in the grasshopper limb (Bentley and Keshishian, 1982; Ho and Goodman, 1982). In these embryos, specific recognition between pioneer filopodia and "landmark" cells, which are interspersed between the sensory axon and the CNS, have been hypothesized to guide pioneer axons (Ho and Goodman, 1982; Goodman et al., 1982; Raper et al., 1983; Taghert et al., 1982). From the array of filopodia extended from a pioneer growth cone, only those that recognize a landmark cell do not retract, and thus the growth cone extends to the landmark cell. As landmark cells are only spaced 50 to $100 \mu \mathrm{m}$ from each other and are within "filopodial grasp" of the pioneer axon, it has been hypothesized that the grasshopper pioneer axon may maneuver through the limb mesenchyme by stepping from one landmark cell to another. In parallel to these studies, we see that clonally related mesodermal cells are within 30 to $50 \mu \mathrm{m}$ of the pioneer motor axon as it leaves the Xenopus neural tube; and an array of such myotubes are scattered along the axon pathway. It is possible that the primary motor axon establishes its peripheral path by stepping from one clonally related myotube to another. However, if the required specific recognition between axon and myotubes was compartmental rather than clonal, the distance over which an axon would have to search for a compatible myotube would be much shorter (namely, to any myotube in the posterior-ventral compartment) than the observed distances between labeled (namely, clonally related) myotubes.

We hypothesize that the compartmental affiliations of other cells may be recognized by the first axons (pioneers) entering peripheral tissues. Later differentiating axons, regardless of their clonal origin or compartmental membership, need only grow along the pioneered pathway, and their selection of specific targets while en route could depend on other features of the environment. This hypothesis suggests that the compartmental organization present in the pregastrula (Jacobson, 1982, 1983) may govern the formation of primary pathways and first connections between CNS and periphery, but it allows other factors to influence the outgrowth of axons at later stages of development. Because the HRP technique allows us to see the initial outgrowth of the axon, the presence and positions of those primary pathways may now be investigated.

\section{References}

Anderson, H. (1981) Projections from sensory neurons developing at ectopic sites in insects. J. Embryol. Exp. Morphol. 65: $209-224$

Bate, C. M. (1976) Pioneer neurons in an insect embryo. Nature 260: 54-56.

Bentley, D., and H. Keshishian (1982) Pathfinding by peripheral pioneer neurons in grasshopper. Science 218: 1082-1088.

Blackshaw, S. E., and A. E. Warner (1976) Low resistance junctions between mesoderm cells during development of trunk muscles. J. Physiol. (Lond.) 255: 209-230.

Blight, A. R. (1978) Golgi-staining of "primary" and "secondary" motoneurons in the developing spinal cord of an amphibian. J. Comp. Neurol. 180: 679-690. 
Coghill, G. E. (1913) The primary ventral roots and somatic motor column of Amblystoma. J. Comp. Neurol. 23: 121-143.

Collins, F., and J. E. Garrett (1980) Elongating nerve fibers are guided by a conditioned medium factor bound to the culture substratum. Proc. Natl. Acad. Sci. U. S. A. 77: 6226-6228.

Crick, F. H. C., and P. A. Lawrence (1975) Compartments and polyclones in insect development. Science 189:340-347.

Deak, I. I. (1978) Thoracic duplications in the mutant wingless of Drosophila and their effects on muscles and nerves. Dev. Biol. 66: 422-441.

Edwards, J. S. (1982) Pioneer fibers. The case for guidance in the embryonic nervous system of the cricket. In Neuronal Development, N. C. Spitzer, ed., pp. 255-266, Plenum Press, New York.

Farel, P. B., and S. E. Bemelmans (1980) Retrograde labeling of migrating spinal motoneurons in bullfrog larvae. Neurosci. Lett. 18: 133-136.

Forehand, C. J., and P. B. Farel (1982) Spinal cord development in anuran larvae. I. Primary and secondary neurons. J. Comp. Neurol. 209: 386-394.

Garcia-Bellido, A. (1975) Genetic control of wing disc development in Drosophila. CIBA Found. Symp. 29: 161-182.

Garcia-Bellido, A., P. Ripoll, and G. Morata (1973) Developmental compartmentalization of the wing disk of Drosophila. Nature New Biol. 245: 251-253.

Garcia-Bellido, A., P. Ripoll, and G. Morata (1976) Developmental compartmentalization in the dorsal mesothoracic disc of Drosophila. Dev. Biol. 48: 132-147.

Ghysen, A. (1978) Sensory neurones recognize defined pathways in Drosophila central nervous system. Nature 274: 869 872.

Ghysen, A. (1980) The projection of sensory neurons in the central nervous system of Drosophila: Choice of the appropriate pathway. Dev. Biol. 78: 521-541.

Ghysen, A., and I. I. Deak (1978) Experimental analysis of sensory nerve pathways in Drosophila. Wilhelm Roux Arch. Dev. Biol. 184: 273283.

Goodman, C. S., J. A. Raper, R. K. Ho, and S. Chang (1982) Pathfinding by neuronal growth cones in grasshopper embryos. In Developmental Order: Its Origin and Regulation, $\mathrm{S}$. Subtelny and P. B. Green, eds., pp. 275-316, Alan R. Liss, Inc., New York.

Gundersen, R. W. and J. N. Barrett (1979) Neuronal chemotaxis: Chick dorsal root axons turn toward high concentrations of nerve growth factor. Science 206: 1079-1080.

Hamilton, L. (1969) The formation of somites in Xenopus. J. Embryol. Exp. Morphol. 22: 253-264.

Harrison, R. G. (1910) The outgrowth of nerve fiber as a mode of protoplasmic movement. J. Exp. Zool. 9: 787-848.

Hirose, G., and M. Jacobson (1979) Clonal organization of the central nervous system of the frog. I. Clones stemming from individual hlastomeres of the 16-cell and earlier stages. Dev. Biol. 71: 191-202.

Ho, R. K., and C. S. Goodman (1982) Peripheral pathways are pioneered by an array of central and peripheral neurones in grasshopper embryos. Nature 297: 404-406.

Holley, J. A. (1982) Early development of the circumferential axonal pathway in mouse and chick spinal cord. J. Comp. Neurol. 205: 371-382.

Holley, J. A., H. O. Nornes, and M. Morita (1982) Guidance of neuritic growth in the transverse plane of embryonic mouse spinal cord. J. Comp. Neurol. 205: 360-370.

Hughes, A. (1959) Studies in embryonic and larval development in amphibia. II. The spinal motor root. J. Embryol. Exp. Morphol. 7: 128-145.

Jacobson, M. (1980) Clones and compartments in the vertebrate central nervous system. Trends Neurosci. $1: 3-5$.

Jacobson, M. (1981) Rohon-Beard neuron origin from blasto- meres of the 16-cell frog embryo. J. Neurosci. 1: 918-922.

Jacobson, M. (1982) Origins of the central nervous system in amphibians. In Neuronal Development, N. C. Spitzer, ed., pp. 45-99, Plenum Press, New York.

Jacobson, M. (1983) Clonal organization of the central nervous system of the frog. III. Clones stemming from individual blastomeres of the 128-, 256-, and 512-cell stages. J. Neurosci. 3: 1019-1038.

Jacobson, M., and G. Hirose (1978) Origin of the retina from both sides of the embryonic brain: A contribution to the problem of crossing at the optic chiasma. Science 202: 637639.

Jacobson, M., and G. Hirose (1981) Clonal organization of the central nervous system of the frog. II. Clones stemming from individual blastomeres of the $32-$ and 64 -cell stages. J. Neurosci. 1: 271-285.

Katz, M. J., and R. J. Lasek (1978) Eyes transplanted to tadpole tails sends axons rostrally in two spinal cord tracts. Science 199: 202-203.

Katz, M. J., and R. J. Lasek (1979) Substrate pathways which guide growing axons in Xenopus embryos. J. Comp. Neurol. 183: 817-832.

Katz, M. J., R. J. Lasek, and H. J. W. Nauta (1980) Ontogeny of substrate pathways and the origin of the neural circuit pattern. Neuroscience 5: 821-833.

Kullberg, R. W., T. L. Lentz, and M. W. Cohen (1977) Development of the myotomal neuromuscular junction in Xenopus laevis: An electrophysiological and fine-structural study. Dev. Biol. 60: 101-129.

Lamborghini, J. E. (1980) Rohon-Beard cells and other large neurons in Xenopus embryos originate during gastrulation. J. Comp. Neurol. 189: 323333.

Lawrence, P. A. (1975) The structure and properties of a compartment border: The intersegmental boundary in Oncopeltus. CIBA Found. Symp. 29: 3-23.

Lawrence, P. A. (1978) Compartments and the insect nervous system. Zoon 6: 157-160.

Letourneau, P. C. (1982) Nerve fiber growth and its regulation by extrinsic factors. In Neuronal Development, N. C. Spitzer, ed., pp. 213-254, Plenum Press, New York.

Mantel, N. (1963) Chi-square tests with one degree of freedom; extensions of the Mantel-Haenszel procedure. J. Am. Stat. Assoc. 58: 690-700.

Mantel, N., and W. Haenszel (1959) Statistical aspects of the analysis of data from retrospective studies of disease. J. Natl. Cancer Inst. 22: 719-748.

Morata, G., and P. A. Lawrence (1977) Homoentic genes, compartments and cell determination in Drosophila. Nature 265: $211-216$.

Morata, G., and P. A. Lawrence (1978) Cell lineage and homeotic mutants in the development of imaginal discs of Drosophila. In The Clonal Basis of Development, S. Subtelny and I. M. Sussex, eds., pp. 45-60, Academic Press, Inc., New York.

Muntz, L. (1964) Neuromuscular foundations of behavior in early stages of the anuran, Xenopus laevis. Doctoral dissertation, University of Bristol, Bristol, England.

Muntz, L. (1975) Myogenesis in the trunk and leg during development of the tadpole of Xenopus laevis. J. Embryol. Exp. Morphol. 33: 757-774.

Nieuwkoop, P. D., and J. Faber (1967) Normal Table of Xenopus laevis (Daudin), Ed. 2, Elsevier North-Holland, Amsterdam.

Nordlander, R. H., J. F. Singer, R. Beck, and M. Singer (1982) An ultrastructural examination of early ventral root formation in Amphibia. J. Comp. Neurol. 199: 535-551.

Palka, J. (1982) Genetic manipulation of sensory pathways in Drosophila. In Neuronal Development, N. C. Spitzer, ed., pp. 121-170, Plenum Press, New York. 
Palka, J., P. A. Lawrence, and H. S. Hart (1979) Neural projection patterns from homeotic tissue of Drosophila studied in bithorax mutants and mosaics. Dev. Biol. 69: 549-575.

Palka, J., M. Schubiger, and H. S. Hart (1981) The path of axons in Drosophila wings in relation to compartment boundaries. Nature 294: 447-449.

Ramón y Cajal, S. (1908) Nouvelles observations sur l'evolution des neuroblastes, avec quelques remarques sur l'hypothese neurogenetique de Hensen-Held. Anat. Anz. 32: 1-25.

Ramón y Cajal, S. (1960) Studies in Vertebrate Neurogenesis, Charles C Thomas, Springfield, IL.

Raper, J. A., M. Bastiani, and C. S. Goodman (1983) Pathfinding by neuronal growth cones in grasshopper embryos. I. Divergent choices made by the growth cones of sibling neurons. J. Neurosci. 3: 20-30.

Roberts, A., J. A. Kahn, S. R. Soffe, and J. D. W. Clarke (1981) Neural control of swimming in a vertebrate. Science 213 : 1032-1034.

Singer, M., R. H. Nordlander, and M. Egar (1979) Axonal guidance during embryogenesis and regeneration in the spinal cord of the newt; the blueprint hypothesis of neuronal pathway patterning. J. Comp. Neurol. 185: 1-22.

Speidel, C. C. (1933) Studies of living nerves. II. Activities of ameboid growth cones, sheath cells and myelin segments, as revealed by prolonged observation of individual nerve fibers in frog tadpoles. Am. J. Anat. 52: 1-79.

Stehouwer, D. J., and P. B. Farel (1980) Central and peripheral controls of swimming in anuran larvae. Brain Res. 195: 323335 .

Stocker, R. F., and P. A. Lawrence (1981) Sensory projections from normal and homoeotically transformed antennae in Drosophila. Dev. Biol. 82: 224-237.

Taghert, P. H., M. Bastiani, R. K. Ho, and C. S. Goodman (1982) Guidance of pioneer growth cones: Filopodial contacts and coupling revealed with an antibody to Iucifer Yellow. Dev. Biol. 94: 391-399.

Taylor, A. C. (1943) Development of the innervation pattern in the limb bud of the frog. Anat. Rec. 87: 379-413.

Vandervorst, P., and A. Ghysen (1980) Genetic control of sensory connections in Drosophila. Nature 286: 65-67.

Weiss, P. (1941) Nerve patterns: The mechanics of nerve growth. Third Growth Symp. 5: 163-203. 